\title{
Síndrome de Moebius, comunicación interventricular asociado a exposición prenatal a misoprostol
}

\author{
HARRY PACHAJOA L. ${ }^{1}$, CAROLINA ISAZA DE L. ${ }^{2}$ \\ 1. MD, PhD en Ciencias Biomédicas énfasis en genética. Centro de Investigaciones en Anomalías Congénitas y \\ Enfermedades Raras (CIACER), Universidad Icesi, Colombia. \\ 2. MD, MSc en morfología énfasis en genética. Universidad del Valle, Colombia.
}

\begin{abstract}
Moebius syndrome, ventricular septal defect due to prenatal exposure to misoprostol

Introduction: Moebius syndrome/sequence is characterized by facial and abducens nerve damage and may be associated with congenital orofacial and limb defects. Additionally, in the last two decades, a possible association with prenatal exposure to misoprostol has been reported. Objective: To present a case of Moebius Syndrome with complex heart disease (ventricular septal defect and pseudocoarctation of the aorta) associated with prenatal exposure to misoprostol. Case report: A 5 year old patient diagnosed with Moebius Syndrome who consulted specialists due to psychomotor retardation, craniofacial, heart and limb defects, and with a history of prenatal exposure to misoprostol is presented. Conclusions: Although the etiology of this syndrome is not clear, hypoxia is a pathophysiological mechanism involved, which can be secondary to prenatal exposure to misoprostol.
\end{abstract}

(Key words: Moebius syndrome, misoprostol, teratogenicity, pseudocoarctation of aorta, ventricular septal defect).

Rev Chil Pediatr 2013; 84 (4): 425-428

\section{RESUMEN}

Introducción: El síndrome o secuencia de Moebius se caracteriza por la afectación del nervio facial y nervio abducens y puede estar asociado a defectos congénitos orofaciales y de las extremidades. Adicionalmente en las dos últimas décadas se han reortada una posible asociación con exposición prenatal a misoprostol. Ob-

Recibido el 04 de agosto de 2012, devuelto para corregir el 20 de noviembre de 2012, segunda versión 8 de marzo de 2013 , tercera versión 15 de abril de 2013, aceptado para publicación el 29 de julio de 2013.

Este trabajo cumple con los requisitos sobre consentimiento /asentimiento informado, comité de ética, financiamiento, estudios animales y sobre la ausencia de conflictos de intereses según corresponda.

Correspondencia a:

Harry Pachajoa, MD, PhD

E-mail: hmpachajoa@icesi.edu.co 
jetivo: Presentar un caso de síndrome de Moebius con cardiopatía compleja (comunicación interventricular y pseudocoartación de aorta) asociado a exposición prenatal a misoprostol. Caso clínico: Paciente de 5 años quien consulta por antecedente de retardo en el desarrollo psicomotor, anomalías craneofaciales, cardiacas y de las extremidades, con antecedente de exposición prenatal a misoprostol, a quien se le diagnóstica síndrome de Moebius. Conclusiones: Aunque la etiología de este síndrome no es clara, un mecanismo fisiopatológico involucrado es el de la hipoxia que puede ser secundario a la exposición prenatal a misoprostol.

(Palabras clave: Síndrome de Moebius, misoprostol, teratogénesis, pseudocoartación de aorta, comunicación interventricular.).

Rev Chil Pediatr 2013; 84 (4): 425-428

\section{Introducción}

El síndrome o secuencia de Moebius (OMIM 157900) es un síndrome polimalformativo que afecta principalmente el nervio facial (VII nervio craneal) y nervio abducens (VI nervio craneal). El fenotipo es variable y puede incluir defectos congénitos orofaciales y de las extremidades ${ }^{1}$.

El mecanismo fisiopatológico no es claro, pero se han propuesto dos teorías: la genética y la isquémica o vascular. En la teoría genética se ha documentado patrones de herencia autosómico dominante, y ligado al $\mathrm{X}$ recesivo, y se ha propuesto un locus localizado en el cromosoma 13 (13q12.2-13)², y en la teoría isquémica o vascular la disminución del flujo sanguíneo y hemorragia y/o muerte celular de las células del núcleo craneal en el área craneal en los núcleos 6 y 7 o una necrosis en estas áreas generarían las características de la disfunción de los pares craneales ${ }^{3}$. Dicha disminución del flujo vascular puede ser secundario a la exposición a misoprostol en el primer trimestre de gestación.

Con el objetivo de ampliar el espectro de los defectos congénitos del síndrome de Moebius y de su posible relación con un evento disruptivo secundario al misoprostol, se presenta un caso de síndrome de Moebius con cardiopatía compleja asociado a exposición prenatal a misoprostol durante el primer trimestre de gestación.

\section{Caso clínico}

Paciente de sexo femenino de 5 años quien consultó por retardo en el desarrollo psicomo- tor, y del lenguaje y defectos congénitos asociados. Producto de primer embarazo de padres no consanguíneos. Como antecedente la madre refirió uso de misoprostol en dosis de $400 \mathrm{mg}$ por vía oral y $200 \mathrm{mg}$ vía vaginal a las 6 semanas de gestación, sin presentar sangrado vaginal. Peso al nacimiento de $2.150 \mathrm{~g}$ y talla $49 \mathrm{~cm}$.

Al examen físico se encontró pliegue epicántico bilateral, parálisis facial bilateral de origen central, microtia bilateral, microsomia hemifacial derecha, macrostomia con desviación de la comisura oral hacia la derecha, filtrum plano, mal oclusión dental, sindactilia cutánea completa del primero y segundo dedo y del tercer y cuarto dedo hasta la falange media de la mano izquierda, en los pies se encontró sindactilia cutánea con compromiso del primer y segundo ortejo bilateral y del tercer y cuarto ortejo la falange proximal del pie izquierdo (figuras 1 y 2). Las medidas antropométricas actuales incluyen perímetro cefálico de $49 \mathrm{~cm}$, peso de $10 \mathrm{~kg}$ y talla de $91 \mathrm{~cm}$, todas por debajo del percentil 5 .

Como estudios complementarios se reporta un cariotipo bandeo $\mathrm{G}$ de alta resolución reportado como normal $(46, \mathrm{XX})$ y ecocardiograma que muestra una comunicación interventricular y pseudocoartación de aorta, ecografía abdominal normal, resonancia cerebral informada como normal, aunque no se realizó uno específicamente para núcleos basales. Por las características mencionadas se hace diagnóstico de síndrome de Moebius.

La madre firma consentimiento informado para la toma de fotografías y su publicación en revistas médicas reservando la identidad del paciente. 


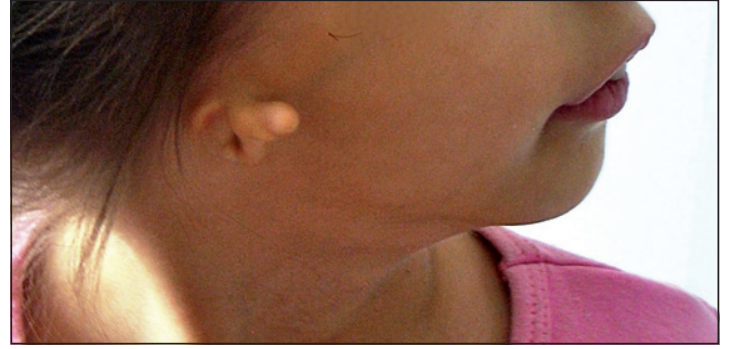

Figura 1. Nótese la microtia y la desviación de la comisura.

\section{Discusión}

La asociación del síndrome de Moebius con otros defectos por disrupción vascular, apoya la teoría vascular. Bouwes-Bavinck y Weaver en 1986 propusieron una teoría unificadora para explicar los hallazgos del síndrome de Moebius, el síndrome de Poland y la secuencia de Klippel Feil. Ellos sugirieron que la interrupción del desarrollo de la arteria subclavia y sus afluentes, (incluida la basilar, las arterias vertebrales y torácica interna, que irrigan el cerebro, el cuello, los músculos pectorales y las extremidades superiores), en o alrededor de la semana sexta intrauterina, es la causal ${ }^{4}$. Se ha documentado la presencia de secuencia de
Moebius y síndrome de Poland en un mismo paciente con exposición prenatal a misoprostol, confirmando un posible origen causado por un disruptor vascular. Adicionalmente se postula que, en el síndrome de Moebius, la regresión de la arteria primitiva del trigémino, que abastece el cerebro posterior antes de la generación de la arteria vertebral o basilar, puede afectar el desarrollo de los núcleos del nervio ${ }^{5}$.

Otros defectos por disrupción vascular asociados se incluyen la gastrosquisis, donde la arteria probablemente involucrada es la arteria onfalomesentérica ${ }^{6}$, y se ha documentado la presencia en un mismo paciente de hasta cuatro diferentes defectos por disrupción vascular (síndrome de Moebius, síndrome de Poland, secuencia de Klippel Feil y síndrome de Goldenhar) ${ }^{7}$.

El misoprostol es un análogo sintético de la prostaglandina E1 aprobado según las entidades reguladoras de medicamentos de muchos países para la prevención y el tratamiento de úlceras gástricas asociadas con el uso de antinflamatorios no esteroideos, por su efecto antisecretor de ácidos gástricos, adicionalmente aumenta la contractilidad uterina por lo que es usada como abortivo ${ }^{8}$.

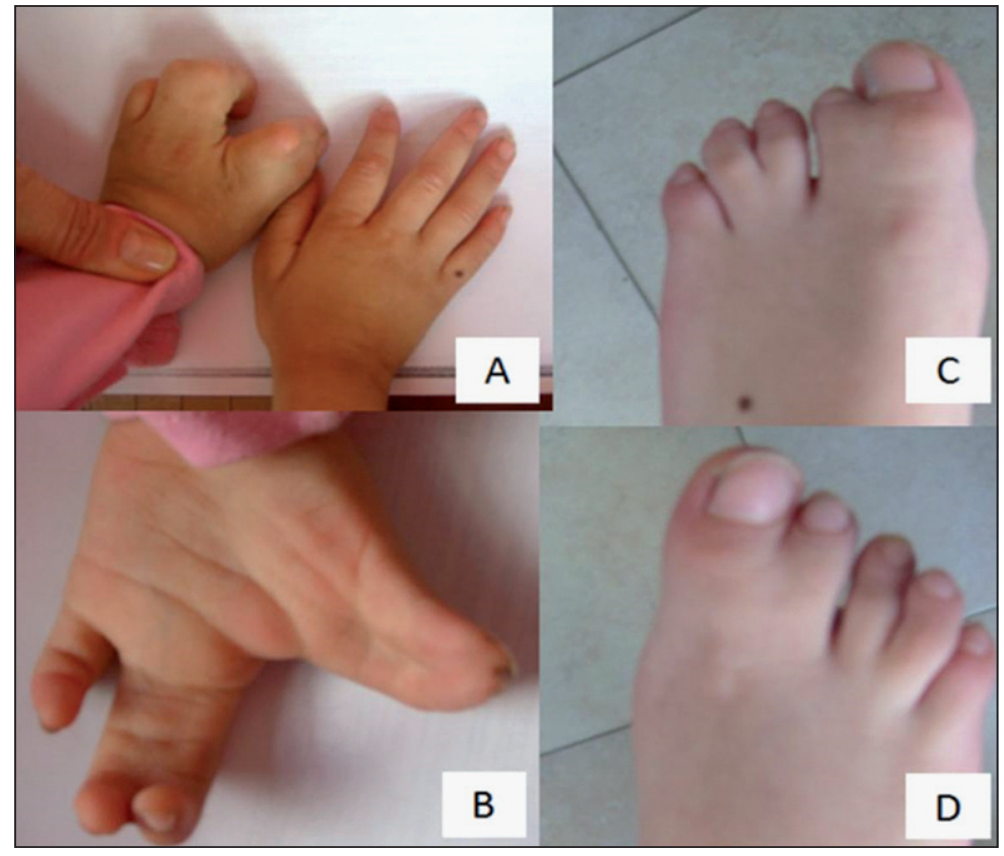

Figura 2. A y B Sindactilia en mano izquierda con fusión del primero y segundo dedo y tercero y cuarto dedo. $\mathrm{C}$ y D, sindactilia en pie, con compromiso del primer y segundo artejo. 
La exposición prenatal a misoprostol se ha asociado a la ocurrencia de defectos por disrupción vascular, principalmente el síndrome de Moebius y defectos de las extremidades de tipo terminal y transversal ${ }^{9,10}$. Otros defectos congénitos asociados al síndrome de Moebius incluye el epicanto interno, la microftalmía, la microtia, la micrognatia, la aplasia o la hipoplasia unilateral del músculo pectoral mayor, la amelia, la sindactilia, la secuencia de Poland, el pie equino varo y la escoliosis ${ }^{11}$. La presentación del síndrome de Moebius y cardiopatía es una asociación poco frecuente ${ }^{12,13}$, pero la presentación de este síndrome con comunicación interventricular y pseudoacoartación de la aorta en un mismo paciente puede sugerir un evento disruptivo común.

Otras entidades de disgenesia oromandibular y de extremidades a tener en cuenta en la paciente son la microsomia hemifacial, el síndrome de Goldenhar entre otros, que podrían corresponder a los diagnósticos diferenciales ${ }^{14}$.

Finalmente, se puede concluir del presente caso que las anomalías que generan un síndrome de Moebius y la cardiopatía compleja pueden sugerir una relación causal con la exposición prenatal a misoprostol secundario a un fenómeno de disrupción vascular. Debido a la asociación de anomalias congénitas relacionadas con exposición prenatal a misoprostol se recomienda una actuación por parte de las instituciones de salud con el fin de prevenir este tipo de eventos, principalmente en paises donde el aborto es ilegal.

\section{Referencias}

1.- Verzijl HT, Padberg GW, Zwarts MJ: The spectrum of Moebius syndrome: an electrophysiological study. Brain 2005; 128: 1728-36.

2.- Kremer H, Kuyt LP, van den Helm B, et al: Localization of a gene for Moebius syndrome to chromosome $3 \mathrm{q}$ by linkage analysis in a Dutch family. Hum Molec Genet 1996; 5: 1367-71.

3.- Shepard TH: Mobius syndrome after misoprostol: a possible teratogenic mechanism. Lancet 1995, 346 (8977): 780 .

4.- Bouwes Bavinck JN, Weaver DD: Subclavian artery supply disruption sequence: Hypothesis of a vascular etiology for Poland, Klippel-Feil, and Mobius anomalies. Am J Med Genet 1986; 23: 903-18.

5.- Deda G, Caksen H, Atalay S: Mobius syndrome associated with ventricular septal defect. Indian J Pediatr 2001; 68 (5): 455-6.

6.- Brockmann K, Backes H, Auber B, Kriebel T, Stellmer $F$, Zoll B: Overlap of Moebius and oromandibular limb hypogenesis syndrome with gastroschisis and pulmonary hypoplasia. Am J Med Genet A 2009; 149A (12): 2832-7.

7.- Cares C, Aravena T: Asociación Sindromática: Poland, Goldenhar, Moebius, Klippel-Feil. Presentación de un Caso Clínico. Rev Chil Pediatr 2010; 81 (1): 53-57.

8.- Norman JE, Thong KG, Baird DT: Uterine contractility and induction of abortion in early pregnancy by misoprostol and mifepristone. Lancet 1991; 338: 1233-6.

9.- Vargas FR, Schuler-Faccini L, Brunoni D, et al: Prenatal exposure to misoprostol and vascular disruption defects: a case- control study. Am J Med Genet 2000; 95: 302-6.

10.- Isaza C, Saldarriaga W, Pachajoa H: Uso inadecuado de misoprostol. ¿Un problema de salud pública? Colomb Med 2008; 39 (2): 61-5.

11.- Pachajoa H, Isaza C: Primer caso de síndrome de Moebius-Poland en niño expuesto prenatalmente a misoprostol. Neurología 2011; 26: 502-3.

12.- Suvarna J, Bagnawar M, Deshmukh CT: Moebius syndrome with total anomalous pulmonary venous connection. Indian J Pediatr 2006; 73: 427-9.

13.- Thapa $R$, Bhattacharya A: Moebius syndrome with atrial septal defect. Singapore Med J 2009; 50 (10): 1030-1.

14.- Hennekam RCM, Krantz ID, Allanson JE (eds): Gorlin's Syndromes of the Head and Neck $5^{\text {th }}$ ed. New York: Oxford University Press; 2010. p. 913-23. 